\title{
Heterogeneous/particle-laden blast waves
}

\author{
D. L. Frost ${ }^{1}$
}

Published online: 2 May 2018

(C) Springer-Verlag GmbH Germany, part of Springer Nature 2018

\section{Overview}

High-speed multiphase flows containing shock or blast waves occur in a wide variety of natural and engineered systems as well as across various length scales. At astrophysical scales, multiphase instabilities driven by shock waves have been cited as the mechanism for the evolution of the turbulent structure of supernova remnants [1,2]. At terrestrial scales, depressurization of a volcanic fluid can lead to explosive volcanic activity associated with a rapid release of gases that drive a blast wave followed by an ash particle flow, as in the case of the eruption of Mt. St. Helens [3]. Vulcanian eruptions, characterized by discrete explosions and shock formation, have been observed to coincide with the formation of dense gas-particle jets with a finger-like structure [4]. At laboratory scales, instabilities in shock-driven multiphase flows play a key role in laser-driven inertial confinement fusion experiments [5].

Shock-driven multiphase flows are a concern in the loss prevention industry, particularly regarding the accidental explosion of combustible dust-air mixtures or hybrid systems containing reactive powder and combustible gases [6]. In commercial or military explosive systems, reactive solid particles may be mixed within an explosive to enhance the explosive performance, which may be characterized by the blast overpressure, the blast impulse, the thermal signature, or the work done by the expanding detonation products [7]. Heat transfer from the burning particles will locally raise the temperature and pressure of the ambient gas, and if the relative velocity between the particles and gas is supersonic,

D. L. Frost

david.frost@mcgill.ca

1 Mechanical Engineering Department, McGill University, 817 Sherbrooke Street West, Montreal, QC H3A 0C3, Canada shock waves may be generated. Embedding inert particles within an explosive or surrounding the explosive with a layer of particles or liquid generally leads to a mitigation in the blast overpressure and impulse, at least in the near field, with a corresponding transfer of kinetic energy to the particles or liquid [8]. As a result of the significant momentum flux associated with the particles, the complex gas-particle flow that is generated may impart a significant impulsive loading on nearby structures or personnel [9]. The experimental and numerical investigation of the properties of such particleladen flows with blast waves is the subject of this thematic issue.

The physical phenomena that must be addressed to predict the behavior of these flows span a range of scales. At the scale of the particles, which may range from microns to millimeters, it is necessary to quantify the effect of particle-particle collisions, particularly in the dense particle regime. The transient forces and heat transfer between particles and the flow during shock-particle interactions must be specified [10]. Of particular importance is determining the rate of relaxation of the relative velocity between the particles and gas behind the shock wave [11]. For reactive particles, the particle reaction rate, which may be limited by kinetics or diffusion, should be quantified, as well as the collective effects from multiple particles [12]. Also, in the case of liquid droplets, droplet fragmentation and vaporization effects may be important. Other effects play a role at scales from millimeters to centimeters and include the development of force-chain networks during the compaction of granular media [13], as well as the subsequent formation of filamentary particle clusters and jets [14]. Finally, at large, integral scales (tens of centimeters to meters), particle-structure and shock-structure interactions come into play as well as shock-shock interactions. 

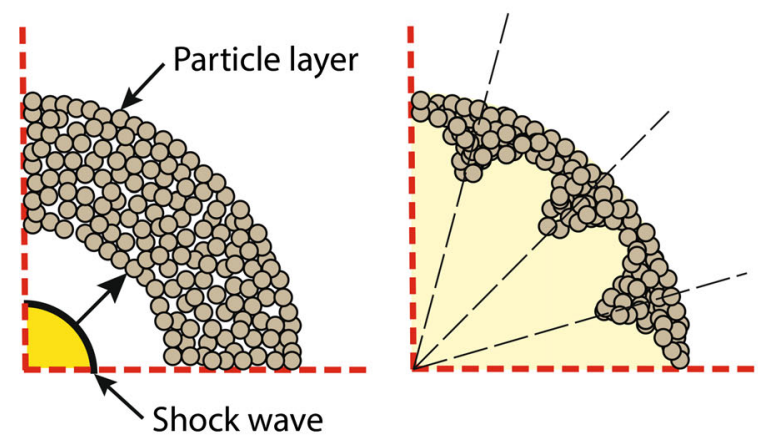

Fig. 1 Schematic of the instability that develops on the interface of the particle layer for a circular ring of particles dispersed by a weak shock wave (adapted from the schematic in Fig. 6 of Rodriguez et al. [15] based on their experiments of the impulsive dispersal of fine particles
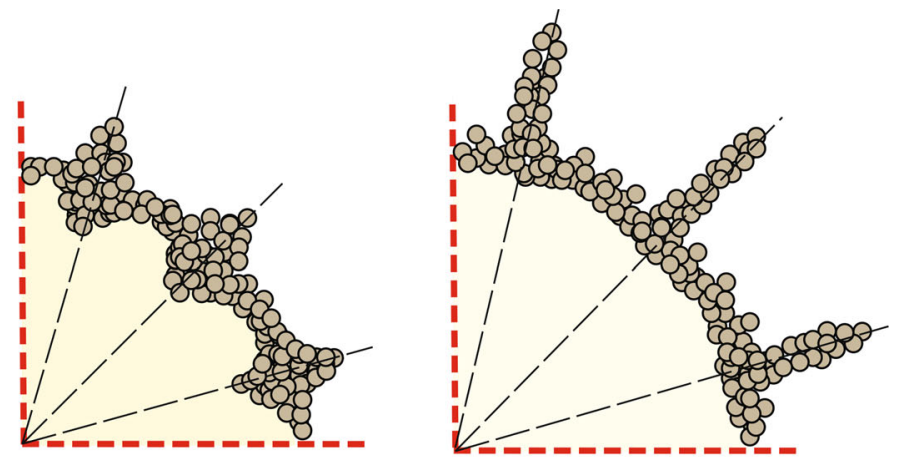

contained within a 2D Hele-Shaw cell). Note that the perturbations initially develop on the inner boundary of the particle layer. The particle clusters catch up to and penetrate the outer portion of the powder layer to form the particle jets observed experimentally

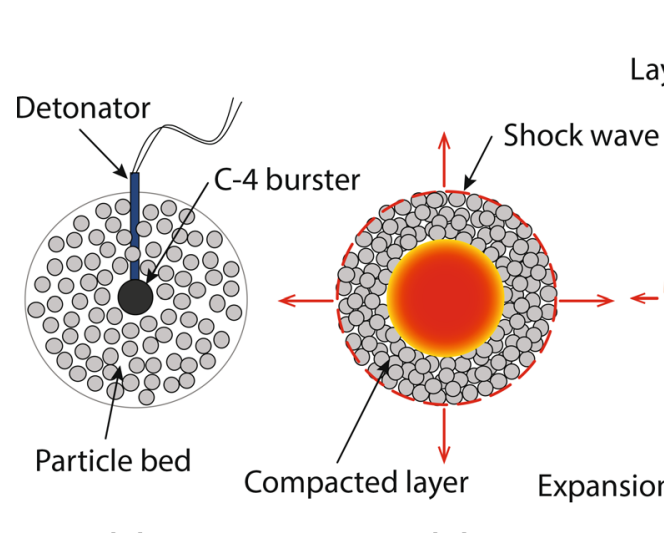

(a)

(b)
Fig. 2 Schematic of the events that occur following the detonation of a high-explosive charge surrounded by a layer of particles, as shown in $\mathbf{a}$. Once the shock reaches the layer-air interface (b), a blast wave is transmitted into the surrounding air and rarefaction waves propagate back into the compacted layer (c), expanding the layer and causing it to frac-

Recent experiments by Wagner et al. [16] within a multiphase shock tube have provided a well-characterized dataset for the validation of the physical processes that occur during the shock interaction with a dense array of particles in air. Ling et al. [17] have demonstrated that the processes associated with the shock interaction with a dense particle curtain differ significantly from those in a dilute gas-particle mixture. In particular, they have shown that for dense particle clouds, important effects to account for during the shock interaction include the two-way coupling between the particles and gas, the particle inertia, and unsteady forces. When a planar shock wave interacts with a planar particle layer within a shock tube, the thickness of the particle layer increases in the longitudinal direction, whereas the layer surfaces remain relatively stable in the transverse direction $[16,17]$. In contrast, systems involving the interaction between a decaying blast wave and a cylindrical or spherical particle layer are more susceptible to the generation of instabilities at the surfaces of the particle layer. Examples of such systems include the dispersal of a circular ring of particles confined between two plates by a radially expanding shock wave (see Fig. 1) and the explosive dispersal of a layer of particles surrounding a spherical or cylindrical high-explosive charge (see Fig. 2). The characteristics of the instabilities that develop in each of these systems are described in the sections below.

A common feature of explosively driven gas-particle flows is the formation of a non-uniform particle distribution which can take the form of particle filaments or jet-like structures. The mechanisms that cause the formation of spatial non-uniformities in the particle number density, for the case of a shock interacting with a particle layer, can be divided broadly into two classes. The first consists of a mul- 


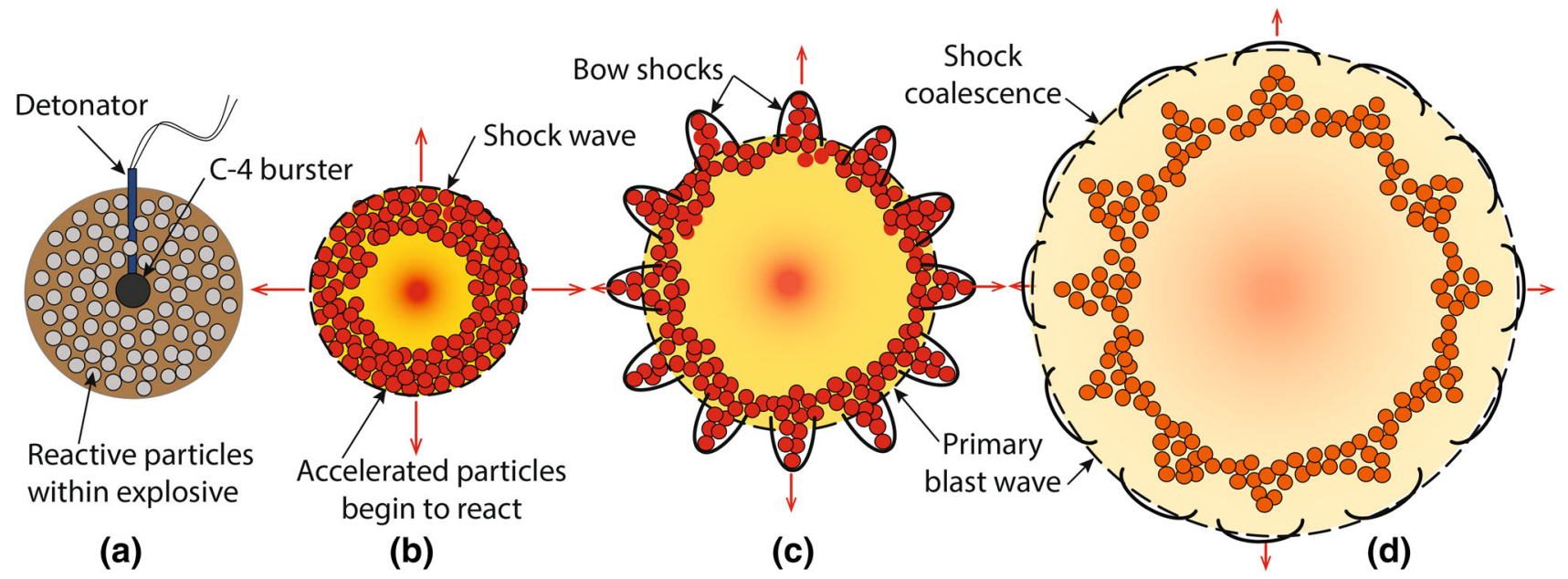

Fig. 3 Schematic of the events that are hypothesized to occur following the detonation of a high-explosive charge with embedded reactive particles, as shown in a. The passage of the detonation wave over the particles and flow behind the detonation rapidly accelerates the particles, and the particles begin to react within the detonation products. When the detonation reaches the outer layer of the explosive (b), a blast wave is transmitted into the surrounding air. As the blast wave expands radi-

tiphase hydrodynamic instability, which is often denoted a shock-driven multiphase instability (SDMI). This interfacial instability mechanism is observed particularly during the interactions between a weak shock and the interfaces of a gas-particle region. This instability is related to the classical hydrodynamic instabilities associated with either the continuous or impulsive acceleration of density interfaces in single-phase flows (i.e., the Rayleigh-Taylor and RichtmyerMeshkov instabilities, respectively), yet has unique features due to the multiphase nature of gas-particle flows in which the transfer of momentum and energy between the phases may occur due to relative motion between the phases.

A second class of mechanisms that lead to non-uniformities in shock-driven gas-particle flows typically occurs during the acceleration of particles, or liquids, with a high explosive. In the case of a stratified layer of particles surrounding a highexplosive charge, the shock transmitted from the explosive into the particle bed has an overpressure that is orders of magnitude greater than shock pressures typically encountered in shock tube studies. The strong shock interacting with the particle layer compacts the layer, causing inelastic deformation of the particles and leading to a bed density approaching that of a solid. The release wave that forms at the outer surface of the layer and propagates inwards, expands the compacted material putting it into tension, which then fractures into large fragments. The radial motion of these fragments, which shed unconsolidated particles, forms the jet-like structures typically observed.

A third class of mechanisms that leads to the formation of particle jets, which appears to be distinct from the above ally and decelerates, the particles, which self-aggregate into conically shaped jets, first cross the detonation products interface (not shown in the figure) and react aerobically in the shocked air. They then penetrate the blast wave and drive bow shocks into the quiescent fluid (c). As the particle jets slow due to drag, the bow shocks run ahead and merge, and eventually, the primary blast wave, coming from behind, coalesces with the bow shocks (d)

two mechanisms, has been observed to occur when light, reactive particles embedded within a liquid or solid explosive are explosively dispersed. In this case, the sequence of events that is hypothesized to occur is shown schematically in Fig. 3. The particles are directly accelerated by the passage of the detonation wave and reach speeds that are a substantial fraction of the velocity of the detonation products on a timescale of the order of nanoseconds. The particles collectively form conically shaped jets, which have a sufficient velocity to penetrate the blast wave that is transmitted into the surrounding atmosphere. The particle jets, moving at a supersonic speed relative to the quiescent gas, drive conical bow shocks. As the particle jets slow, the bow shocks move ahead of the particle jets and coalesce, and eventually, the primary blast wave, moving in the shocked air perturbed by the bow shocks, reaches and merges with the bow shocks.

The three mechanisms noted above that lead to the formation of particle jets or filaments during explosive particle dispersal will be explored in more detail in the following section, with illustrations provided from experimental investigations in each case.

\section{Particle jet formation}

Schematics of the two mechanisms for shock destabilization of the surfaces of a layer of particles are given in Figs. 1 (for a weak shock) and 2 (for a strong shock). The formation of particle jets for the case of particles embedded within a high explosive is shown schematically in Fig. 3. These three mechanisms are described in more detail in the sections below. 


\subsection{Shock-driven multiphase instability}

The instability that occurs when weak shocks interact with gas-particle mixtures is closely related to the RichtmyerMeshkov instability (RMI) [19,20], which occurs when a density interface in a fluid is impulsively accelerated by the interaction of a shock wave with the interface. The instability is associated with the generation of vorticity at the interface due to misalignment of the interfacial density and pressure gradients. A density interface may also be formed if a cloud of solid or liquid particles is dispersed within a gas. Vorobieff et al. [21] and Anderson et al. [22] have clarified the relationship between SDMI and RMI with experimental and computational studies. Their experiments examined the shock interaction with a gas column seeded with droplets. They found that the interaction of the shock with the cylindrical droplet volume generated counter-rotating vortices in a similar manner as for the single-phase case of a shock in air interacting with a cylindrical volume of heavy gas [23]. They pointed out that while in both cases vortices are formed, in the single-phase case, the density change occurs across a distinct interface, whereas for the multiphase case, gradients of average density play a role. They also noted that the physical mechanism associated with the multiphase analog of the RMI differs from the classical baroclinic mechanism in that after the passage of the shock, the particle (or droplet) velocity can lag behind that of the gas.

Ukai et al. [24] carried out a numerical and analytical study of the instability that develops in shock-driven gas-particle mixtures. They considered a planar shock wave interacting with a gas-particle mixture with a sinusoidal perturbation imposed on the interface of the two-phase region. They found that the growth rate of the perturbation predicted with the multiphase model, for the case of very small Stokes numbers (St $\ll 1)$, agreed well with the classical single-phase RMI growth rates. Very small particles subject to a shock accommodate sufficiently rapidly with the ambient flow so that the two-phase mixture effectively acts like a dense gas. For larger particles, where St is on the order of unity or a larger value $(S t \geq 1)$, the particles do not experience an impulsive acceleration, but instead accelerate continuously, so that the instabilities grow exponentially, similar to the behavior observed in the Rayleigh-Taylor instability (RTI).

A convenient arrangement for visualizing the instabilities that develop when a layer of particles is dispersed by a shock is the 2D Hele-Shaw apparatus used by Rodriguez et al. [15,25,26], as well as, more recently, by Xue et al. [27]. They attached an air shock tube perpendicular to the center of a Hele-Shaw cell containing a ring of fine powder. When the radial shock wave passed through the powder, they observed low-wavenumber filamentary perturbations growing on the inner surface of the powder layer and high-wavenumber jets appearing later on the outer surface of the layer. The growth

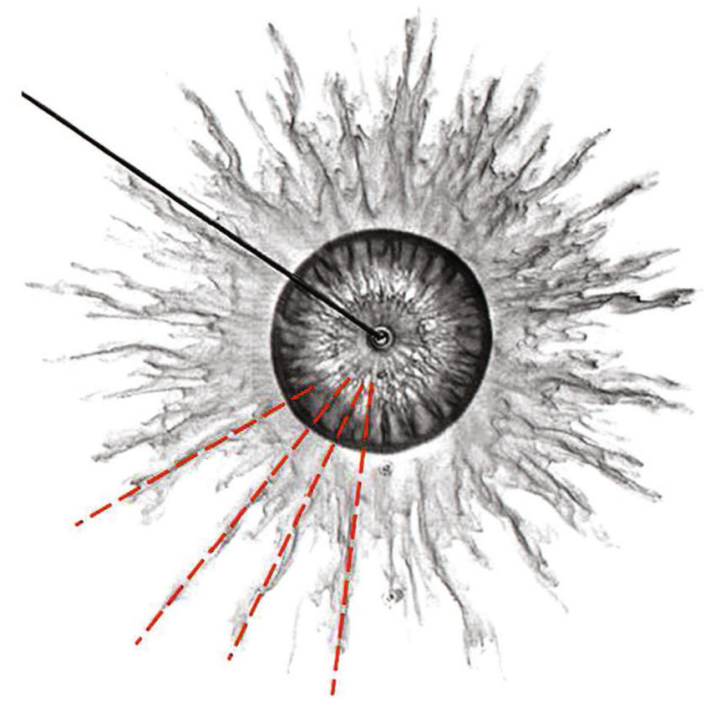

Fig. 4 Dispersal of a ring of fine powder within a Hele-Shaw cell with a shock wave. The picture shows a superposition of two images of the powder, taken at times of 6 and $57 \mathrm{~ms}$. The particle filaments visible on the inner surface of the particle layer at the earlier time are correlated with the particle jets visible at the later time. (Jets are identified with the dashed red lines.) Taken from Fig. 7 of [15] with permission

of the large inner perturbations is consistent with the SDMI, since perturbations on the inner surface subject to an outgoing shock wave are subject to unstable growth. Rodriguez et al. [15] demonstrated, with an examination of their highspeed video results, that the particle filaments that develop initially on the inner surface, but are moving outwards, eventually penetrate the remainder of the powder layer to form the outward moving particle jets that are observed at later times. An example of the jet formation they observed is shown in Fig. 4, which shows a superposition of two images, illustrating the correlation between the early-time internal particle filaments and the late-time external jets. This sequence of events is also shown schematically in Fig. 1, which illustrates the development of the particle jets following the shockparticle layer interaction. Through an extensive series of experiments, Rodriguez et al. [15] also found that the wave number of the particle jets increases with shock overpressure and with decreasing particle density.

To increase the strength of the shock impinging on a particle layer, it is convenient to utilize a quantity of high explosives. However, it is difficult to design a 2D HeleShaw experiment with high explosives, unless the Hele-Shaw plates are sufficiently massive to reduce the out-of-plane motion. One method is to partially constrain the motion of a ring of powder with layers of dense steel beads, as in the experiments of Frost et al. [14]. However, it is more convenient to study the development of instabilities during the dispersal of a powder layer by a high-explosive charge in a 1D spherical geometry. The development of perturbations to 

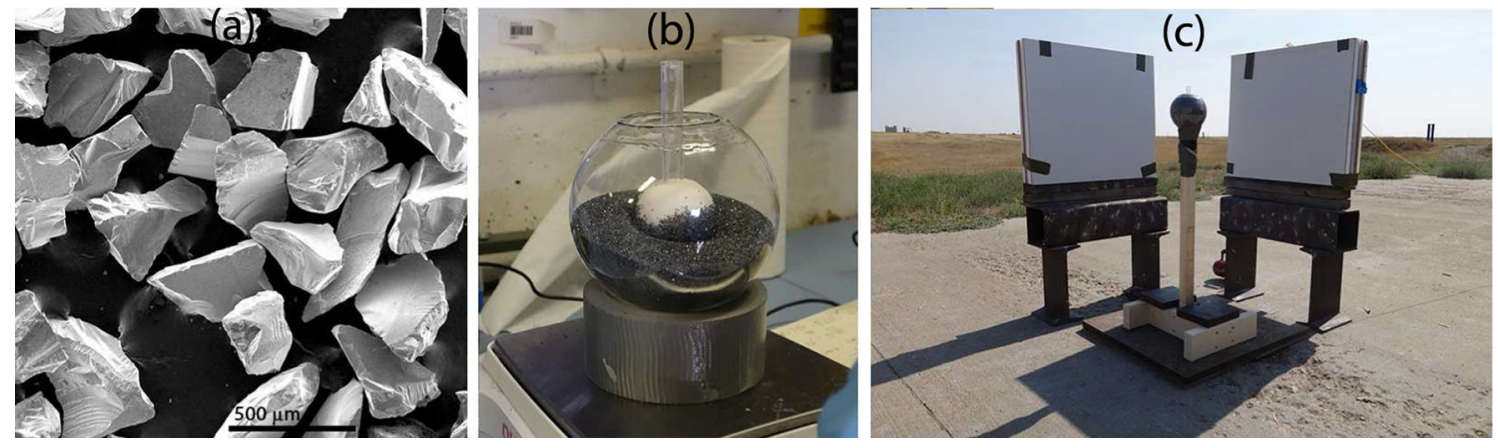

Fig. 5 Composite of images related to the explosive dispersal trial shown in Fig. 6. a SEM image of $\mathrm{SiC}$ powder used in the trial, b photograph of spherical glass casing partially filled with $\mathrm{SiC}$ powder, with central ball of C-4, $\mathbf{c}$ photograph of the charge placed on top

the particle density field during explosive particle dispersal is described in the next section.

\subsection{Shock-compaction-fracture-driven particle jetting}

A schematic of the events that occur during the dispersal of a bed of particles with a high-explosive charge in spherical geometry is shown in Fig. 2, as first proposed by Milne et al. [28]. The initial charge shown in Fig. 2a consists of a spherical bed of packed solid particles, with a spherical ball of C-4 explosive positioned at the center of the charge. After detonation of the explosive, a shock wave travels radially outwards through the particle bed and eventually reaches the outer surface of the particle bed (Fig. 2b). At that point, a blast wave is transmitted into the surrounding air and a release wave travels inwards through the compacted powder, causing it to expand radially outwards (Fig. 2c). Circumferential tension within the particle bed induces the propagation of cracks through the partially consolidated powder. Empirically, it is observed that the spacing of the fragments that are generated, $\delta$, is on the order of the thickness of the compacted layer at the time when the expansion wave reaches the inner surface of the particle bed. The fragments move radially outwards, shedding unconsolidated powder, forming jet-like structures (Fig. 2d). Depending on the degree of consolidation of the parent fragments, the parent fragments may completely shed their initial core, at which point the radial jet motion stops.

Milne et al. [28] observed with radiography that the nascent formation of the perturbations to the particle bed that develop into the particle jets observed at later times occurred on the same timescale as the transit time for the shock to reach the outer surface of the bed and the expansion wave to then reach the inner bed surface. They concluded that the jet formation is related to the fracture of the compacted particle bed, rather than the growth of a hydrodynamic instability, which occurs over much longer timescales [29]. As an example, to illustrate the time at which perturbations form within the particle bed following the passage of the shock, of a wooden post on the test site, with two protected X-ray film cassettes visible behind the charge. Photographs taken at the Flash X-ray test site at DRDC Suffield, Alberta, Canada (unpublished)

Figs. 5 and 6 show the experimental arrangement and results for the explosive dispersal of a packed bed of brittle silicon carbide particles $(\mathrm{SiC})$. The charge (shown partially filled with powder in Fig. 5b) consists of a glass sphere containing $1661 \mathrm{~g}(M)$ of $\mathrm{SiC}$ with a centrally located $90 \mathrm{~g}(C)$ ball of C-4 $(M / C=18.5)$. The $\mathrm{SiC}$ particles (30 grit with a nominal size of about $400 \mu \mathrm{m}$ ) have an irregular shape as shown in the SEM image in Fig. 5a. The arrangement on the test site is shown in Fig. 5c, which shows two X-ray cassettes, placed perpendicular to the charge. Figure 6 shows single frames taken from the high-speed video record of the explosive dispersal process, as well as the two radiographs taken during the experiment. The first two video images correspond closely to the times of the radiographs. At the time of the first video image ( $140 \mu$ s after detonation), the outer surface of the expanding particle layer appears smooth, although the corresponding radiograph (taken at about $146 \mu \mathrm{s}$ ) indicates that the particle distribution is highly non-uniform at this time. The perturbations are formed at an earlier time, likely on the order of the time for one wave transit within the particle bed, estimated to be about $50 \mu \mathrm{s}$. The second radiograph (taken at about $296 \mu$ s after detonation) shows that all the $\mathrm{SiC}$ particles are grouped into clusters, with a bimodal size distribution. Each of the larger clusters visible on the radiograph corresponds to a compacted fragment of $\mathrm{SiC}$ particles which moves out radially, shedding individual $\mathrm{SiC}$ particles, forming the billowing wakes visible in the fourth video frame. When the parent fragments have been completely exhausted, the particle jets reach their maximum radial extent. The large jets visible have considerable momentum and the impact of the jets with the layer of particle board protecting the front surface of the X-ray cassette is sufficient to punch holes in the plate, as visible in Fig. 7. Note that the impact of the minor fragments visible in radiograph shown in Fig. $6 \mathrm{f}$ is also visible on the witness plate shown in Fig. 7, but they have insufficient momentum to penetrate the plate. Although the mechanism for the formation of the particle jets (Fig. 2) appears to be related to a brittle fragmentation mechanism, a 


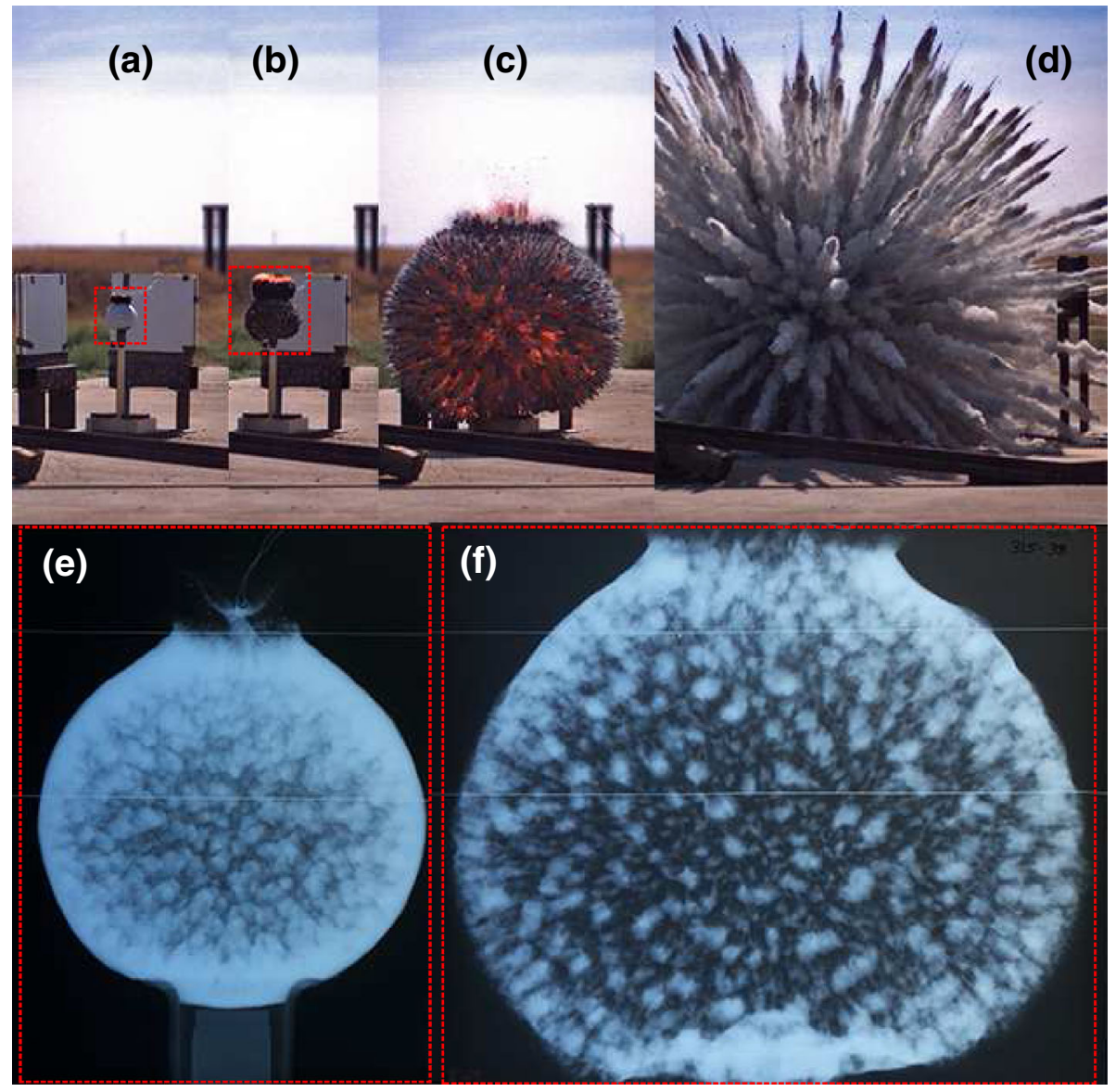

Fig. 6 Single frames from the high-speed video record of the explosive dispersal of $\mathrm{SiC}$ particles. Times of the frames are a $140 \mu \mathrm{s}$, b $300 \mu \mathrm{s}$, c $1780 \mu \mathrm{s}$, d $5980 \mu \mathrm{s}$. e Radiograph taken at about the same time $(146 \mu \mathrm{s})$ as the video image shown in $\mathbf{a}$. Similarly, $\mathbf{f}$ is the radiograph taken at about the same time $(296 \mu \mathrm{s})$ as the video image in $\mathbf{b}$. The non-uniform particle clusters visible in the radiographs move rapidly radially outwards and shed unconsolidated particles, producing the jet-

detailed model is not yet available to predict the size distribution of the fragments or the dependence on material properties, which will influence the degree of shock consolidation of the material.

\subsection{Detonation-driven particle jetting}

The jet formation that can occur when light reactive particles embedded within a high explosive are explosively dispersed is shown schematically in Fig. 3. (Note that heavy embedded particles, such as steel particles, typically do not form jets during explosive dispersal [30].) The jet formation mechanism differs from the above mechanisms in several important aspects. First, since the particles are embedded within the explosive with no inter-particle voids, compaction of a particle layer does not occur. Instead, the passage of the detonation wave over the particles rapidly accelerates the particles to a velocity which is a substantial fraction of the radial veloc- like structures visible in image (d). A photograph of one of the particle board sheets that protect the front of the X-ray cassettes, taken after the trial, is shown in Fig. 7. Experiments carried out at the Flash Xray test site at DRDC Suffield, Alberta, Canada (unpublished results by D. L. Frost, J. Loiseau, S. Goroshin, and F. Zhang, 2017, trial FX315-38, used with permission)

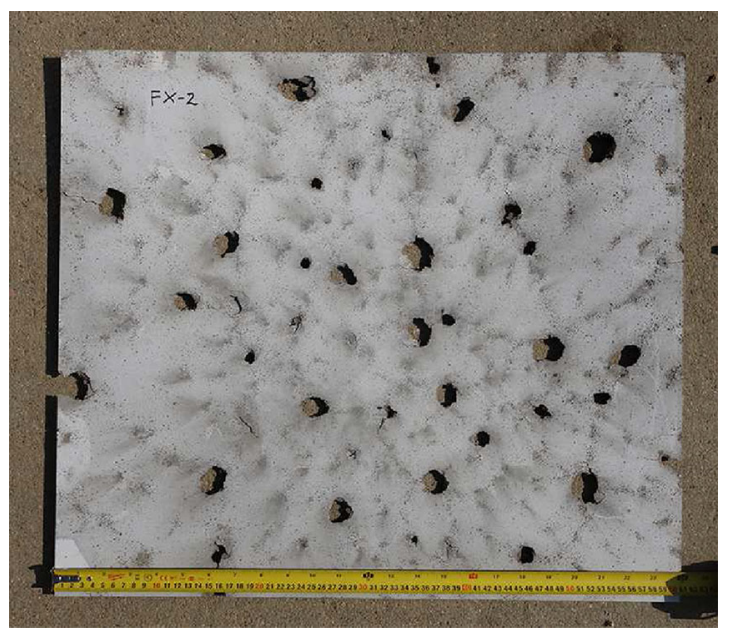

Fig. 7 Post-test photograph of one of the particle board sheets that cover the X-ray cassettes and act as witness plates during the experimental trial shown in Fig. 6, illustrating the effect of the impact of the particle jets. Witness plate initially located about $55 \mathrm{~cm}$ from center of charge 

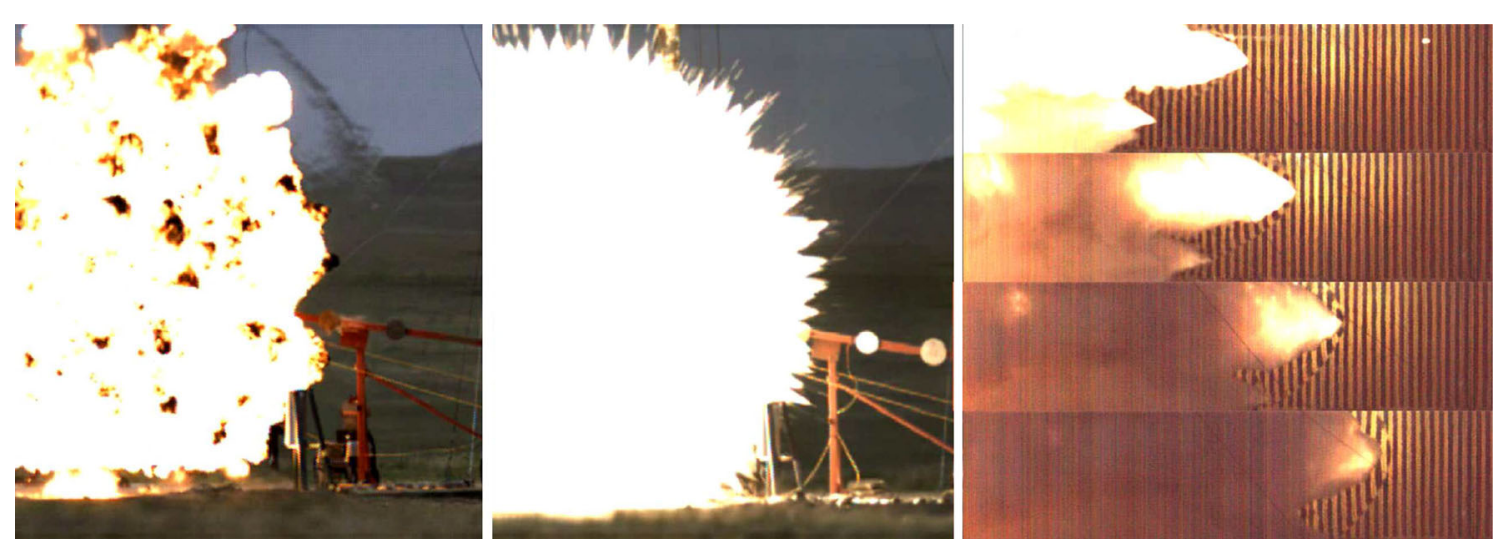

Fig. 8 Comparison of the development of the fireball surface morphology from homogeneous and heterogeneous explosive charges (adapted from [32]): (left) fireball from homogeneous C-4 charge illustrating the billowing perturbations that develop on the detonation product interface as a result of the Rayleigh-Taylor instability. A strong blast wave is visible outside the fireball near the top of the photo; (middle) fireball from an improvised explosive formulation comprised of oxidizer, metallic particles, and a non-energetic fuel, exhibiting the formation of reactive particle jets. Note that no blast wave is visible ahead of the jets; (right) close-up of the motion of a reactive particle jet that moves ahead of the primary blast wave from a heterogeneous charge. The bow shock driven by the particle jet, visible against the zebra board backdrop, begins to detach from the jet and becomes less curved as the jet slows. The parameters of the tests are given in [33] (left, trial 0; middle, trial 8; right, trial 19)

surface structure. The mechanism which leads to the formation of the jets has not yet been definitively articulated, and, for example, it is not clear whether the interaction between the particles and the unsteady fluid motion associated with the RTI plumes at the product interface plays a role in the jet formation. The series of four video images shown on the right of Fig. 8, taken from another trial with an explosive charge containing metal particles, exhibits the motion of one of the metal particle jets and the bow shock that is driven ahead of the jet. In these images, there is no primary blast wave visible ahead of the jets. However, at later times, as the particle jets slow due to drag, the multiple bow shocks move ahead of the particle jets and merge together. The initial shock wave generated at the surface of the explosive, which

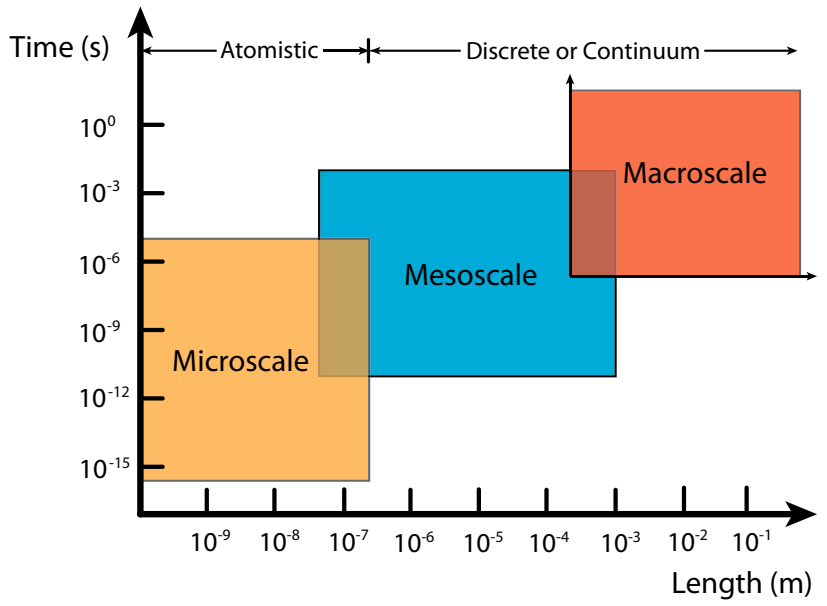

Fig. 9 Physical and temporal scales associated with modeling regimes Adapted from [50] 
Table 1 Multiphase flow modeling approaches Adapted from [51]

\begin{tabular}{|c|c|c|c|c|c|}
\hline Type of model & Model name & Gas phase & Solid phase & Gas-solid coupling & Scale \\
\hline Atomistic & Molecular dynamics & Lagrangian & Lagrangian & $\begin{array}{l}\text { Elastic collisions at particle } \\
\text { surface }\end{array}$ & Micro \\
\hline Discrete & $\begin{array}{l}\text { Smoothed particle } \\
\text { hydrodynamics }\end{array}$ & Lagrangian & Lagrangian & Short-range potential & Macro \\
\hline Continuum & $\begin{array}{l}\text { Resolved discrete } \\
\text { particle model }\end{array}$ & Eulerian (resolved) & Lagrangian & $\begin{array}{l}\text { Boundary condition at } \\
\text { particle surface }\end{array}$ & Macro \\
\hline Continuum & $\begin{array}{l}\text { Unresolved discrete } \\
\text { particle model }\end{array}$ & $\begin{array}{l}\text { Eulerian } \\
\quad \text { (unresolved) }\end{array}$ & Lagrangian & Gas-particle drag closure & Macro \\
\hline Continuum & Two-fluid model & Eulerian & Eulerian & Gas-solid drag closure & Macro \\
\hline
\end{tabular}

lags behind the particle jets at the time of the images shown on the right of Fig. 8, eventually reaches and coalesces with the bow shocks.

\section{Computational approaches}

A variety of computational models have been used to investigate the interactions of shock waves within multiphase flows. The modeling of heterogeneous explosive systems involves a range of length- and timescales, as shown schematically in Fig. 9. Here the term microscale refers to length- (and time-) scales at which atomistic and molecular effects play a role. At the so-called mesoscale, phenomena that occur at the scale of the particles (ranging typically from $0.1 \mu \mathrm{m}$ to $1 \mathrm{~mm}$ ) play an important role. The largest scale, or macroscale, is associated with the size of the simulation domain.

The types of modeling approaches associated with the various scales are listed in Table 1. At the microscale, Lagrangian models based on ab initio molecular dynamics (MD) are commonly used to investigate the material dynamics and reaction, particularly for energetic materials. MD is computationally intensive and limited to computational domains that are typically much smaller than for problems of engineering interest. Hence, most multiphase engineering models assume that one (or both) of the phases can be considered to be a continuum.

An exception to the continuum assumption is the smoothed particle hydrodynamics (SPH) method, in which the materials are discretized into a set of particles, which interact through a short-range potential. Initially developed for the simulation of astrophysical phenomena, the SPH method is a meshless method that has the advantage of being able to handle extreme interface deformations and density variations. SPH has been used to simulate interfacial multiphase fluid flows (e.g., [35]), as well as the specific problem of the explosive dispersal of particles [36]. The SPH method yields qualitatively reasonable results, although the choice of appropriate material models to produce quantitatively accurate predictions remains largely unresolved.
The majority of engineering models for simulating heterogeneous/particle-laden blast waves are based on one of the continuum approaches listed in Table 1 . Most models are based on a solution of the Euler equations, although there is currently a significant effort being applied within the computational community to incorporate turbulence effects in modeling compressible multiphase flows (e.g., [37]). With continuum models, while the conservation laws governing the multiphase flow are known, defining the closure terms specifying the exchange of mass, momentum, and energy between the phases remains a significant challenge $[38,39]$. Furthermore, to close the equations for the multiphase flow, it is necessary to introduce an additional condition related to the compressibility of each phase and the compaction of the granular bed, as discussed by Baer and Nunziato [40].

The simplest continuum approach for modeling particleladen compressible flows is the two-fluid model listed as the last item in Table 1. Also referred to as the dusty gas model $[41,42]$, this model assumes that the particles are sufficiently small that they perfectly follow the local gas flow. Hence, the particle-laden gas can be considered to be a single fluid where the density depends on the local particle number density. For most particle sizes of interest, this assumption is too restrictive and the relative motion between the particles and ambient fluid must be considered. In the next level of complexity (fourth model listed in Table 1), the particles are treated as a separate phase in a Lagrangian manner, with the interstitial gas treated as another phase mapped onto an Eulerian grid. In this case, the particles are not explicitly resolved, and the momentum coupling between the phases is treated by specifying a gas-particle drag closure. Depending on the problem considered and the nature of the particles, the exchange of heat and mass (due to particle reaction or evaporation) may also be considered. In this formulation, the interaction between a shock or detonation wave with individual particles cannot be resolved explicitly. Hence, the interactions occur at a sub-grid scale and separate models must be specified for the heat and momentum transfer that occurs 
during the shock-particle or detonation-particle interaction [31].

The third model listed in Table 1 refers to the case in which individual particles are explicitly resolved. In this model, the multibody particle collisions can be modeled directly as well as the non-steady shock-particle and particle-flow interactions. Modeling a large number of particles is computationally intensive, with current exascale computations limited to tracking on the order of $10^{4}$ particles [43]. Since this is still much less than the number of particles in typical experimental systems (e.g., a 1-L sphere maximally packed with 10- $\mu$ m-diameter particles contains on the order of $10^{11}$ particles), a common technique is to have a single computational particle represent a large number of physical particles.

Examples of the use of the two continuum models listed in Table 1 in which the solid phase is treated in a Lagrangian manner are represented by papers in this thematic issue [4449].

\section{Thematic issue on heterogeneous/particle-laden blast waves}

The objective of the current thematic issue is to bring together experimental and computational researchers working in the area of compressible multiphase flows driven by shock or blast waves. Some issues that need to be addressed by the shock physics community include a clear articulation of the mechanisms responsible for the formation of interfacial instabilities in gas-particle flows. Ideally, a model should lead to quantitative predictions of the scale and growth rate of perturbations that develop at the surface of particle layers subject to acceleration by a shock wave. Some progress is being made, particularly for the case of a weak shock interacting with a particle cloud, as exemplified by the papers in this thematic issue. However, for strong shocks associated with high explosives, an additional complication is the particle deformation that occurs at these extreme pressures. During the explosive dispersal of particles (or liquids), the exchange of momentum and energy between the phases plays a key role in the decay of the blast wave overpressure and impulse. Both inert and reactive particles may mitigate, or augment, the blast wave, depending on these exchange terms, and the prediction of particle-blast interaction is clearly important from the point of view of applications. Computation of compressible multiphase flows remains a difficult task, with the choice of the closure of the multiphase model by specifying the interaction between the phases a particular challenge. The role of turbulence in a multiphase flow remains an open question. All of the issues mentioned above are addressed by the papers in this thematic issue, which are briefly summarized in the next section.
Note that every contributed paper to this thematic issue has been rigorously peer reviewed, following the established policies and procedures of the Shock Waves Journal. Each paper was reviewed by two or more independent referees, each an internationally recognized expert in the field. Of the 13 papers submitted for the thematic issue, nearly equally divided between experimental and numerical contributions, one paper was withdrawn following a "major revisions" decision, and the 12 papers were accepted after typically two rounds of revision.

\subsection{Overview of papers in thematic issue}

The interaction of a weak shock wave with a particle layer is addressed in several computational studies. Osnes et al. [44] develop a model to investigate particle jetting observed by Rodriguez et al. [15] during the shock acceleration of a cylindrical shell of particles confined within a Hele-Shaw cell. They systematically vary the gas-solid coupling mechanism to determine the primary mechanism responsible for jet formation. Black et al. [45] also present numerical simulations of a shock-driven multiphase instability, by considering the interaction of a shock wave with a circular region dilutely seeded with particles. They consider the effect of different particle force models on the development of the instability.

Several papers consider the multiphase flow generated by the explosive dispersal of inert, solid (or liquid) particles. Experimentally, Loiseau et al. [18] focus on the terminal velocity attained by the accelerated material, extracted from high-speed images, and compare the results with predictions from the conventional Gurney model as well as a modified version. They quantify the differences in the predictions for solid particles and liquids, as compared with the experimental results. In a related study, Pontalier et al. [52] examine the same experiments to extract the blast overpressure and impulse to determine the influence of the interaction between the particles and the flow and blast wave. In a companion manuscript, Pontalier et al. [46] use a multiphase hydrocode to explore the effect of various parameters on the particleblast interaction which influences the decay rate of the blast wave overpressure and impulse.

McGrath et al. [53] present numerical predictions for the flow generated during shock-driven particle dispersal for initial conditions ranging from a packed particle bed to a dilute particle suspension. They demonstrate good agreement with experiments ranging from particle motion driven by a shock within a shock tube, to particles dispersed with a high explosive. Ling and Balachandar [47] computationally investigated the spherical particle-laden blast wave generated by the sudden release of a spherical volume of a high-pressure gas-particle mixture. By carrying out a scaling analysis, they find that a particular length scale may be used to approximately collapse the results over a wide range of pressure 
ratios. Mo et al. [48] use a discrete formulation to simulate the explosive dispersal of a finite number of particles by directly tracking the motion of individual particles and particle-particle collisions.

Sen et al. [49] have critically examined the closure terms for gas-solid momentum and energy transfer that are needed in macroscale models of shock-particle interactions. Their work represents the first attempt to quantify the effect of turbulent velocity fluctuations for systems involving shockparticle interactions.

One paper in this thematic issue considers the dispersal and reaction of reactive particles and droplets. In a large-scale experimental study, Bai et al. [54] investigate the detonation of large droplet clouds generated by the explosive dispersal of a metalized slurry. The dispersal process exhibits jet formation, although casing fracture effects may influence the jet scale.

The manuscript by Rigby et al. [55] investigates the explosive dispersal of saturated soils from a buried explosive charge, with the motivation of protecting personnel and structures against buried landmines and IEDs. By directly measuring the pressure and impulse from the buried explosive, they found a single fundamental loading mechanism, independent of particle size and soil cohesion.

The only technical note in the thematic issue has been contributed by Sugiyama et al. [56] who carried out twodimensional experiments in which an explosive charge was buried within a sand hill in the shape of a triangular prism. They studied the mitigation of the peak blast wave overpressure and impulse by the sand.

Acknowledgements The editors would like to thank the authors for their enthusiastic response to the call for papers and their important contributions to the theme of this thematic issue. We would like to thank all the reviewers for providing valuable comments and questions that significantly improved the manuscripts. We thank the editors assigned to each of the papers, with special thanks to Andrew Higgins for dedicating part of his sabbatical to pushing this issue toward completion. Guidance from Evgeny Timofeev and Harald Kleine regarding the technical management of this issue is much appreciated. The author of this editorial would also like to acknowledge Fan Zhang of DRDC Suffield for support of the unpublished work shown in Fig. 6, as well as the contributions of Scott Trebble (photography) and Steve Mowers (radiography) to the experiments.

\section{References}

1. Miles, A.R.: The blast-wave-driven instability as a vehicle for understanding supernova explosion structure. Astrophys. J. 696, 498-514 (2009). doi:10.1088/0004-637X/696/1/498

2. Inoue, T., Yamazaki, R., Inutsuka, S.-I.: Turbulence and magnetic field amplification in supernova remnants: interactions between a strong shock wave and multiphase interstellar medium. Astrophys. J. 695, 825-833 (2009). doi:10.1088/0004-637X/695/2/825

3. Kieffer, S.W.: Blast dynamics at Mount St Helens on 18 May 1980. Nature 291, 568-570 (1981). doi:10.1038/291568a0
4. Formenti, Y., Druitt, T.H., Kelfoun, K.: Characterisation of the 1997 Vulcanian explosions of Soufriere Hills Volcano, Montserrat, by video analysis. Bull. Volcanol. 65, 587-605 (2003). doi:10.1007/ s00445-003-0288-8

5. Aglitskiy, Y., Vilikovich, A.L., Karasik, M., Metzler, N., Zalesak, S.T., Schmitt, A.J., Phillips, L., Gardner, J.H., Serlin, V., Weaver, J.L., Obenschain, S.P.: Basic hydrodynamics of RichtmyerMeshkov-type growth and oscillations in the inertial confinement fusion-relevant conditions. Phil. Trans. R. Soc. A 368, 1739-1768 (2010). doi:10.1098/rsta.2009.0131

6. Eckhoff, R.K.: Dust explosion prevention and mitigation, status and developments in basic knowledge and in practical application. Int. J. Chem. Eng. 2009, 569825 (2009). doi:10.1155/2009/569825

7. Frost, D.L., Kleine, H., Slanik, M., Higgins, A., McCahan, S., Zhang, F., Murray, S.B.: Blast waves from heterogeneous explosives. In: Ball, G.J., Hillier, R., Roberts, G.T. (eds.) Proceedings of the 22nd International Symposium on Shock Waves, vol. 2, pp. 947-952. Imperial College, London, 18-23 July (1999)

8. Allen, R.M., Kirkpatrick, D.J., Longbottom, A.W., Milne, A.M., Bourne, N.K.: Experimental and numerical study of free-field blast mitigation. AIP Conf. Proc. 706(1), 823-826 (2004). doi:10.1063/ 1.1780363

9. Frost, D.L., Ornthanalai, C., Zarei, Z., Tanguay, V., Zhang, F.: Particle momentum effects from the detonation of heterogeneous explosives. J. Appl. Phys. 101(11), 113529 (2007). doi:10.1063/1. 2743912

10. Ling, Y., Haselbacher, A., Balachandar, S.: Importance of unsteady contributions to force and heating for particles in compressible flows. Part 1: Modeling and analysis for shockparticle interaction. Int. J. Multiph. Flow 37, 1026-1044 (2011). doi:10.1016/j. ijmultiphaseflow.2011.07.001

11. Boiko, V.M., Kiselev, V.P., Kiselev, S.P., Papyrin, A.N., Poplavskii, S.V., Fomin, V.M.: Interaction of a shock wave with a cloud of particles. Combust. Explos. Shock Waves 32(2), 191-203 (1996). doi:10.1007/BF02097090

12. Soo, M., Goroshin, S., Bergthorson, J.M., Frost, D.L.: Reaction of a particle suspension in a rapidly-heated oxidizing gas. Propellants Explos. Pyrotech. (2015). doi:10.1002/prep.201400269

13. Majmudar, T.S., Behringer, R.P.: Contact force measurements and stress-induced anisotropy in granular materials. Nature 435, 10791082 (2005). doi:10.1038/nature03805

14. Frost, D.L., Gregoire, Y., Petel, O.E., Goroshin, S., Zhang, F.: Particle jet formation during explosive dispersal of solid particles. Phys. Fluids 24(9), 091109 (2012). doi:10.1063/1.4751876

15. Rodriguez, V., Saurel, R., Jourdan, G., Houas, L.: Impulsive dispersion of a granular layer by a weak blast wave. Shock Waves 27(2), 187-198 (2016). doi:10.1007/s00193-016-0658-8

16. Wagner, J.L., Beresh, S.J., Kearney, S.P., Corbin, C., Trott, W.M., Castaneda, J.N., Pruett, B.O., Baer, M.R.: A multiphase shock tube for shock wave interactions with dense particle fields. Exp. Fluids 52, 1507-1517 (2012). doi:10.1007/s00348-012-1272-x

17. Ling, Y., Wagner, J.L., Beresh, S.J., Kearney, S.P., Balachandar, S.: Interaction of a planar shock wave with a dense particle curtain: Modeling and experiments. Phys. Fluids 24, 113301 (2012). doi:10. $1063 / 1.4768815$

18. Loiseau, J., Pontalier, Q., Milne, A.M., Goroshin, S., Frost, D.L.: Terminal velocity of liquids and granular materials accelerated by a high explosive. Shock Waves 28(3) (2018). doi:10.1007/ s00193-018-0822-4

19. Richtmyer, R.D.: Taylor instability in shock acceleration of compressible fluids. Commun. Pure Appl. Math. 13(2), 297-319 (1960). doi:10.1002/cpa.3160130207

20. Meshkov, E.E.: Instability of the interface of two gases accelerated by a shock wave. Fluid Dyn. 4(5), 101-104 (1969). doi:10.1007/ BF01015969 
21. Vorobieff, P., Anderson, M., Conroy, J., White, R., Truman, C.R., Kumar, S.: Vortex formation in a shock-accelerated gas induced by particle seeding. Phys. Rev. Lett. 106, 184503 (2011). doi:10. 1103/PhysRevLett.106.184503

22. Anderson, M., Vorobieff, P., Truman, C.R., Corbin, C., Kuehner, G., Wayne, P., Conroy, J., White, R., Kumar, S.: An experimental and numerical study of shock interaction with a gas column seeded with droplets. Shock Waves 25, 107-125 (2015). doi:10. 1007/s00193-015-0555-6

23. Haas, J.-F., Sturtevant, B.: Interaction of weak shock waves with cylindrical and spherical gas inhomogeneities. J. Fluid Mech. 181, 41-76 (1987). doi:10.1017/S0022112087002003

24. Ukai, S., Balakrishnan, K., Menon, S.: On Richtmyer-Meshkov instability in dilute gas-particle mixtures. Phys. Fluids 22, 104103 (2010). doi:10.1063/1.3507318

25. Rodriguez, V., Saurel, R., Jourdan, G., Houas, L.: Solid-particle jet formation under shock-wave acceleration. Phys. Rev. E 88, 063011 (2013). doi:10.1103/PhysRevE.88.063011

26. Rodriguez, V., Saurel, R., Jourdan, G., Houas, L.: External front instabilities induced by a shocked particle ring. Phys. Rev. E 90, 043013 (2014). doi:10.1103/PhysRevE.90.043013

27. Xue, K., Du, K., Shi, X., Gan, Y., Bai, C.: Dual hierarchical particle jetting of a particle ring undergoing radial explosion. Soft Matter (2018). doi:10.1039/C8SM00209F

28. Milne, A.M., Floyd, E., Longbottom, A.W., Taylor, P.: Dynamic fragmentation of powders in spherical geometry. Shock Waves 24, 501-513 (2014). doi:10.1007/s00193-014-0511-x

29. Milne, A.M., Parrish, C., Worland, I.: Dynamic fragmentation of blast mitigants. Shock Waves 20(1), 41-51 (2010). doi:10.1007/ s00193-009-0235-5

30. Zhang, F., Frost, D.L., Thibault, P.A., Murray, S.B.: Explosive dispersal of solid particles. Shock Waves 10(6), 431-443 (2001). doi:10.1007/PL00004050

31. Ripley, R.C., Zhang, F., Lien, F.-S.: Acceleration and heating of metal particles in condensed matter detonation. Proc. R. Soc. Lond. A: Math. Phys. Eng. Sci. 468(2142), 1564-1590 (2012). doi:10. 1098/rspa.2011.0595

32. Ritzel, D.V., Ripley, R.C., Murray, S.B., Anderson, J.: Near-field blast phenomenology of thermobaric explosions. In: Hannemann, K., Seiler, F. (eds.) Shock Waves, pp. 305-310. Springer, Berlin (2009). doi:10.1007/978-3-540-85168-4_48

33. Murray, S.B., Anderson, C.J., Gerrard, K.B., Smithson, T., Williams, K., Ritzel, D.V.: Overview of the 2005 Northern Lights Trials. In: Hannemann, K., Seiler, F. (eds.) Shock Waves, pp. 335340. Springer, Berlin (2009). doi:10.1007/978-3-540-85168-4_53

34. Ripley, R.C., Donahue, L., Dunbar, T.E., Murray, S.B., Anderson, C.J., Zhang, F., Ritzel, D.V.: Ground reflection interaction with height-of-burst metalized explosions. In: Hannemann, K., Seiler, F. (eds.) Shock Waves, pp. 281-286. Springer, Berlin (2009). doi:10. 1007/978-3-540-85168-4 44

35. Krimi, A., Rezoug, M., Khelladi, S., Nogueira, X., Deligant, M., Ramrez, L.: Smoothed particle hydrodynamics: A consistent model for interfacial multiphase fluid flow simulations. J. Comput. Phys. 358, 53-87 (2018). doi:10.1016/j.jcp.2017.12.006

36. Gregoire, Y., Frost, D.L., Petel, O.: Development of instabilities in explosively dispersed particles. AIP Conf. Proc. 1426, 1623-1626 (2012). doi:10.1063/1.3686596

37. Balachandar, S., Eaton, J.K.: Turbulent dispersed multiphase flow. Annu. Rev. Fluid Mech. 42(1), 111-133 (2010). doi:10.1146/ annurev.fluid.010908.165243

38. Bdzil, J.B., Menikoff, R., Son, S.F., Kapila, A.K., Stewart, D.S.: Two-phase modeling of deflagration-to-detonation transition in granular materials: A critical examination of modeling issues. Phys. Fluids 11(2), 378-402 (1999). doi:10.1063/1.869887
39. Baer, M.R.: Modeling heterogeneous energetic materials at the mesoscale. Thermochim. Acta 384(1-2), 351-367 (2002). doi:10. 1016/S0040-6031(01)00794-8

40. Baer, M.R., Nunziato, J.W.: A two-phase mixture theory for the deflagration-to-detonation transition (DDT) in reactive granular materials. Int. J. Multiph. Flow 12(6), 861-889 (1986). doi:10. 1016/0301-9322(86)90033-9

41. Carrier, G.F.: Shock waves in a dusty gas. J. Fluid Mech. 4(4), 376-382 (1958). doi:10.1017/S0022112058000513

42. Marble, F.E.: Dynamics of dusty gases. Annu. Rev. Fluid Mech. 2(1), 397-446 (1970). doi:10.1146/annurev.fl.02.010170.002145

43. Balachandar, S.: Recent advances in compressible multiphase flows explosive dispersal of particles. In: Future Directions in CFD Research, a Modeling and Simulation Conference, Hampton (2012)

44. Osnes, A.N., Vartdal, M., Pettersson Reif, B.A.: Numerical simulation of particle jet formation induced by shock wave acceleration in a Hele-Shaw cell. Shock Waves 28(3) (2018). doi:10.1007/ s00193-017-0778-9

45. Black, W.J., Denissen, N., McFarland, J.A.: Particle force model effects in a shock-driven multiphase instability. Shock Waves 28(3) (2018). doi:10.1007/s00193-017-0790-0

46. Pontalier, Q., Lhoumeau, M.G., Milne, A.M., Longbottom, A.W., Frost, D.L.: Numerical investigation of particle-blast interaction during explosive dispersal of liquids and granular materials. Shock Waves 28(3) (2018). doi:10.1007/s00193-018-0820-6

47. Ling, Y., Balachandar, S.: Simulation and scaling analysis of a spherical particle-laden blast wave. Shock Waves 28(3) (2018). doi:10.1007/s00193-017-0799-4

48. Mo, H., Lien, F.-S., Zhang, F., Cronin, D.S.: A numerical framework for the direct simulation of dense particulate flow under explosive dispersal. Shock Waves 28(3) (2018). doi:10.1007/ s00193-017-0741-9

49. Sen, O., Gaul, N.J., Davis, S., Choi, K.K., Jacobs, G., Udaykumar, H.S.: Role of pseudo-turbulent stresses in shocked particle clouds and construction of surrogate models for closure. Shock Waves 28(3) (2018). doi:10.1007/s00193-017-0801-1

50. Ripley, R.C.: Acceleration and heating of metal particles in condensed matter detonation. PhD Thesis, University of Waterloo (2010)

51. van der Hoef, M.A., van Sint Annaland, M., Deen, N.G., Kuipers, J.A.M.: Numerical simulation of dense gas-solid fluidized beds: a multiscale modeling strategy. Annu. Rev. Fluid Mech. 40(1), 47-70 (2008). doi:10.1146/annurev.fluid.40.111406.102130

52. Pontalier, Q., Loiseau, J., Goroshin, S., Frost, D.L.: Experimental investigation of blast mitigation and particle-blast interaction during the high-explosive dispersal of particles and liquids. Shock Waves 28(3) (2018). doi:10.1007/s00193-018-0821-5

53. McGrath, T., Clair, J.S., Balachandar, S.: Modeling compressible multiphase flows with dispersed particles in both dense and dilute regimes. Shock Waves 28(3) (2018). doi:10.1007/ s00193-017-0726-8

54. Bai, C.-H., Wang, Y., Xue, K., Wang, L.-F.: Experimental study of detonation of large-scale powder-droplet-vapor mixtures. Shock Waves 28(3) (2018). doi:10.1007/s00193-017-0795-8

55. Rigby, S.E., Fay, S.D., Tyas, A., Clarke, S.D., Reay, J.J., Warren, J.A., Gant, M., Elgy, I.: Influence of particle size distribution on the blast pressure profile from explosives buried in saturated soils. Shock Waves 28(3) (2018). doi:10.1007/s00193-017-0727-7

56. Sugiyama, Y., Izumo, M., Ando, H., Matsuo, A.: Two-dimensional explosion experiments examining the interaction between a blast wave and a sand hill. Shock Waves 28(3) (2018). doi:10.1007/ s00193-018-0813-5 